Gut and Liver, Vol. 9, No. 6, November 2015, pp. 697-698

\title{
Initial Steps to Prevent Nonsteroidal Anti-Inflammatory Drug- or Aspirin- Induced Enteropathy: Long-Term Outcome Data
}

\author{
In Seok Lee and Yu Kyung Cho \\ Department of Internal Medicine, The Catholic University of Korea College of Medicine, Seoul, Korea
}

See "Long-Term Outcomes of NSAID-Induced Small Intestinal Injury Assessed by Capsule Endoscopy in Korea: A Nationwide Multicenter Retrospective Study" by Ki-Nam Shim, et al. on page 727-733, Vol. 9. No. 6, 2015

Nonsteroidal anti-inflammatory drugs (NSAIDs) and lowdose aspirin are among the most frequently prescribed and used medications. ${ }^{1}$ With the development of video capsule endoscopy, the frequency and severity of NSAID enteropathy has become more evident. ${ }^{2}$ NSAID-induced enteropathy has a different pathological mechanism from gastroduodenal mucosal injury, so different treatments and preventative strategies are needed.

NSAID-induced enteropathy is common. Approximately 70\% of regular NSAID users develop injury in the small intestine, which is more common than gastroduodenal mucosal injuries. ${ }^{2}$ Endo et al. $^{3}$ reported small-bowel mucosal breaks in 57.6\% of patients receiving NSAIDs. There is a poor correlation between NSAID-induced damage and symptoms in the small intestine. NSAID-induced enteropathy is usually asymptomatic or has nonspecific symptoms, such as iron-deficiency anemia, occult blood, diarrhea, hypoalbuminemia, and malabsorption of vita$\min \mathrm{B}_{12}$. Endo et al. ${ }^{4}$ studied patients on low-dose aspirin with obscure gastrointestinal (GI) bleeding with capsule endoscopy and found that more than 95\% of the patients had some mucosal injury in the small bowel. The enteropathy was characterized by multiple petechiae and loss of villi. It revealed that even low doses of NSAIDs could lead to the generation of erosions and ulcerations in the small intestine. ${ }^{5}$ Maiden et al. ${ }^{6}$ reported mucosal injury in the small bowel in 68\% to 75\% of volunteers after 2 weeks of therapy with $75 \mathrm{mg}$ slow-release diclofenac taken twice daily.

Recent studies have reported unwanted and more dangerous adverse effects in the small intestine if proton pump inhibitors (PPIs) are combined with NSAIDs. ${ }^{7}$ PPIs, by suppressing gastric acid secretion, are very effective for reducing NSAID-induced damage in the stomach but are without proven benefit in preventing NSAID-related damage in the rest of the GI tract. ${ }^{7}$ Moreover, PPIs alter the microbiome in the small intestine. ${ }^{8}$ Capsule endoscopy studies have revealed that adding PPIs to NSAIDs results in a higher frequency of mucosal lesions in the small intestine than the frequency observed in patients taking NSAIDs alone. ${ }^{9,10}$ Some investigators have reported that all patients taking both NSAIDs and PPIs have ulcerations in the small intestine. ${ }^{9}$ Most patients (80\% to $100 \%$ ) taking low-dose NSAIDs and PPI therapy have active mucosal lesions in the small bowel after 2 weeks of therapy. ${ }^{10}$ A recent prospective capsule endoscopy study found that enteric-coated aspirin and PPI were the two most important risk factors for the development of mucosal breaks in the small intestine. ${ }^{3}$ However, it should be considered that it was an observational cross-sectional study, and PPI use is a well-known marker of comorbidity that might not have been properly controlled for in the analysis. ${ }^{11}$ No human clinical trial has shown that the combination of a PPI and aspirin is associated with an increased risk of lower GI bleeding.

Strategies to prevent aspirin- or NSAID-induced enteropathy include the identification and modification of associated risk factors and co-prescription of a protective agent. ${ }^{12}$ Shim et al. ${ }^{13}$ showed outcomes and long-term follow-up data in a nationwide multicenter study in Korea and reported that recurrence of NSAID-induced small bowel injury was not frequent (4.6\%). Conservative management was also sufficient in most recurrent cases. The risk factors for recurrence were old age and hypertension. However, that study did not present accurate information of coadministered drugs after the initial episode of bleeding because of its retrospective design. However, after or during the

Correspondence to: Yu Kyung Cho

Department of Internal Medicine, Seoul St. Mary's Hospital, The Catholic University of Korea College of Medicine, 222 Banpo-daero, Seocho-gu, Seoul 06591, Korea

Tel: +82-2-2258-6024, Fax: +82-2-2258-2055, E-mail: ykcho@catholic.ac.kr

pISSN 1976-2283 eISSN 2005-1212 http://dx.doi.org/10.5009/gnl15287

() This is an Open Access article distributed under the terms of the Creative Commons Attribution Non-Commercial License (http://creativecommons.org/licenses/by-nc/4.0) which permits unrestricted non-commercial use, distribution, and reproduction in any medium, provided the original work is properly cited. 
first episode of small bowel bleeding, 64\% of patients received PPIs. These patients might have added or continued to take PPIs for treatment of enteropathy alone as well as to lower the risk of upper GI bleeding. Six patients showed recurrence. There were no comparative data on PPI use between the recurrent and nonrecurrent cases. If it is supposed that all recurred cases were taking PPIs, the maximum possible recurrence rate for NSAIDPPI coadministration would be 6 of 61 (10\%). The study did not analyze which type of aspirin the patients had used. However, in Korea, most of the aspirin prescribed for cardiovascular prevention is the enteric coated form. In the initial episode of bleeding, the most prominent capsule endoscopic findings were multiple ulcerations (59\%) and erosions or aphthae (23\%). However, the diagnosis of recurrence depended on clinical findings without a repeated capsule endoscopy. Lesions in the small intestine might tend to persist whether NSAID therapy is continued or not.

NSAID- or low-dose aspirin-induced enteropathy is common but can be asymptomatic. ${ }^{11}$ Moreover, because of the difficulty in diagnosing it, as well as the lack of any proven-effective treatment for NSAID-induced enteropathy, the clinical relevance may be underestimated. Shim et al. ${ }^{13}$ showed a clinically benign course of enteropathy in long-term follow-up data. Further studies of drug interactions and risk factor analyses are required to determine an effective preventive strategy.

\section{CONFLICTS OF INTEREST}

No potential conflict of interest relevant to this article was reported.

\section{REFERENCES}

1. Thomas J, Straus WL, Bloom BS. Over-the-counter nonsteroidal anti-inflammatory drugs and risk of gastrointestinal symptoms. Am J Gastroenterol 2002;97:2215-2219.

2. Graham DY, Opekun AR, Willingham FF, Qureshi WA. Visible small-intestinal mucosal injury in chronic NSAID users. Clin Gastroenterol Hepatol 2005;3:55-59.

3. Endo H, Sakai E, Taniguchi L, et al. Risk factors for small-bowel mucosal breaks in chronic low-dose aspirin users: data from a prospective multicenter capsule endoscopy registry. Gastrointest Endosc 2014;80:826-834.

4. Endo H, Hosono K, Inamori M, et al. Characteristics of small bowel injury in symptomatic chronic low-dose aspirin users: the experience of two medical centers in capsule endoscopy. J Gastroenterol 2009;44:544-549.

5. Endo H, Hosono K, Inamori M, et al. Incidence of small bowel injury induced by low-dose aspirin: a crossover study using capsule endoscopy in healthy volunteers. Digestion 2009;79:44-51.

6. Maiden L, Thjodleifsson B, Theodors A, Gonzalez J, Bjarnason I. A quantitative analysis of NSAID-induced small bowel pathology by capsule enteroscopy. Gastroenterology 2005;128:1172-1178.

7. Marlicz W, Loniewski I, Grimes DS, Quigley EM. Nonsteroidal anti-inflammatory drugs, proton pump inhibitors, and gastrointestinal injury: contrasting interactions in the stomach and small intestine. Mayo Clin Proc 2014;89:1699-1709.

8. Wallace JL, Syer S, Denou E, et al. Proton pump inhibitors exacerbate NSAID-induced small intestinal injury by inducing dysbiosis. Gastroenterology 2011;141:1314-1322.e5.

9. Watanabe T, Sugimori S, Kameda N, et al. Small bowel injury by low-dose enteric-coated aspirin and treatment with misoprostol: a pilot study. Clin Gastroenterol Hepatol 2008;6:1279-1282.

10. Kuramoto T, Umegaki E, Nouda S, et al. Preventive effect of irsogladine or omeprazole on non-steroidal anti-inflammatory druginduced esophagitis, peptic ulcers, and small intestinal lesions in humans, a prospective randomized controlled study. BMC Gastroenterol 2013;13:85.

11. Lanas A, Sostres C. PPI therapy: the small bowel, low-dose aspirin and PPIs. Should we be concerned? Nat Rev Gastroenterol Hepatol 2014;11:458-460.

12. Kim JH, Park SH, Cho CS, et al. Preventive efficacy and safety of rebamipide in nonsteroidal anti-inflammatory drug-induced mucosal toxicity. Gut Liver 2014;8:371-379.

13. Shim KN, Song EM, Jeen YT, et al. Long-term outcomes of NSAID-induced small intestinal injury assessed by capsule endoscopy in Korea: a nationwide multicenter retrospective study. Gut Liver 2015;9:727-733. 